\title{
BMJ Open Association between diabetes-specific health literacy and health-related quality of life among elderly individuals with pre-diabetes in rural Hunan Province, China: a cross- sectional study
}

\author{
Zhao Hu, ${ }^{1}$ Lulu Qin, ${ }^{2}$ Huilan $X{ }^{1}$
}

To cite: Hu Z, Qin L, Xu H. Association between diabetesspecific health literacy and health-related quality of life among elderly individuals with pre-diabetes in rural Hunan Province, China: a crosssectional study. BMJ Open 2019;9:e028648. doi:10.1136/ bmjopen-2018-028648

- Prepublication history for this paper is available online. To view these files, please visit the journal online (http://dx.doi. org/10.1136/bmjopen-2018028648).

Received 18 December 2018 Revised 02 August 2019 Accepted 05 August 2019

A) Check for updates

(C) Author(s) (or their employer(s)) 2019. Re-use permitted under CC BY-NC. No commercial re-use. See rights and permissions. Published by BMJ.

${ }^{1}$ Department of Social Medicine and Health Management, Xiangya School of Public Health, Central South University, Changsha, China

${ }^{2}$ Department of Social Medicine and Health Management, School of Medicine, Hunan Normal University, Changsha, China

Correspondence to Huilan Xu; xhl6363@sina.com

\section{ABSTRACT}

Objectives To examine the association between diabetesspecific health literacy (DSHL) and health-related quality of life (HRQoL) among elderly individuals with pre-diabetes in rural China.

Design, setting and participants This cross-sectional study included 434 elderly individuals with pre-diabetes from 42 villages in rural China.

Main outcome measures HRQoL was assessed using the Medical Outcomes Study 36-Item Short-Form Health Survey. DSHL was measured by a validated questionnaire in China. Differences in HRQoL between groups with and without high DSHL were tested by multivariate analysis of covariance (MANCOVA).

Results The prevalence of pre-diabetes was $21.5 \%$. The average age of participants $(n=434)$ was $69.4 \pm 6.4$ years, and $58.5 \%$ were female. Bivariate analysis showed that those with high DSHL had increases of 2.9 points in the physical health component score and 4.4 points in the mental health component score (MCS) compared with those without. After adjustment for potential confounders, a significant MANCOVA model (Wilks' $\lambda=0.974, F=5.63$, $p=0.004$ ) indicated that individuals with pre-diabetes who had high DSHL reported higher MCS $\left(\mathrm{M}_{\text {diff }}=3.5,95 \% \mathrm{Cl} 1.8\right.$ to 6.3 , effect size $=0.38$ ). This remained significant across subscales: general health $(\mathrm{p}=0.028)$, vitality $(\mathrm{p}=0.014)$, social functioning $(p=0.017)$ and mental health $(p=0.005)$. Conclusions Low DSHL was associated with worsening $\mathrm{HRQOL}$ among elderly individuals with pre-diabetes in rural China, particularly in the mental health components. Trial registration number ChiCTR-IOR-15007033.

\section{INTRODUCTION}

Pre-diabetes describes individuals who have impaired fasting glucose (IFG) or/and impaired glucose tolerance (IGT) ${ }^{1}$ Pre-diabetes is a less common but important condition that constitutes an intermediate state between type 2 diabetes and healthy status. Several studies have identified that individuals with pre-diabetes have a high risk of
Strengths and limitations of this study

- This is the first study to examine the association between health-related quality of life (HRQLL) and diabetes-specific health literacy (DSHL) among elderly individuals with pre-diabetes in rural China.

- The study provides valuable information on $\mathrm{HRQOL}$ among elderly individuals with pre-diabetes in rural areas in China.

- The association between HRQoL and DSHL was analysed in eight domains, as well as in the physical health component and the mental health component, making the results more comprehensive.

- The cross-sectional study design makes causal relationships undeterminable.

developing diabetes, and the occurrence increases with age..$^{2-4}$ Approximately $5 \%-10 \%$ of people with pre-diabetes become diabetic annually, although the progression rate varies by population and the definition of pre-diabetes. ${ }^{5}$ In China, the estimated prevalence of pre-diabetes was $35.7 \%$ in adults and $45.8 \%$ in the elderly population in 2013. ${ }^{7}$ Therefore, people with pre-diabetes, especially elderly, are an important target group for interventions intended to prevent diabetes.

Health-related quality of life (HRQoL) is a comprehensive and multidimensional condition that refers to an individual's perceived physical and mental health under the influence of illness, injury and treatment over time. ${ }^{8}{ }^{9}$ Several studies have demonstrated that many risk factors, such as smoking, chronic diseases, poor diet, insufficient physical activity and overweight, lead to lower HRQoL. ${ }^{10-12}$ Because biomedical measures sometimes may not sensitively indicate the deterioration or 
improvement in symptoms and health status, HRQoL has been increasingly incorporated as a complementary and essential outcome measure in medical interventions and population health surveys to assess changes in the physical, mental and social well-being of these individuals. Studies have found that the HRQoL is usually impaired in individuals with pre-diabetes compared with the healthy population; additionally, individuals with pre-diabetes progressing to diabetes suffer from a great loss in HRQoL. ${ }^{13-15}$ Moreover, HRQoL affects both the entry and subsequent utilisation of health services and the cost of healthcare in China. ${ }^{1617}$ Thus, assessing HRQoL in the intermediate period between normal plasma glucose and type 2 diabetes is important; because the concept has a broader definition that enables us to fully understand both the somatic and emotional health statuses of individuals with pre-diabetes and consequently create interventions to improve it, especially by relieving pain, malaise and consequences of diseases. ${ }^{18}$

Health literacy (HL) is the degree to which individuals have the capacity to obtain, process and understand the basic health information and service need to make informed health decision. ${ }^{19}$ Over the past decades, a growing body of research suggests that inadequate HL is associated with adverse health outcomes, such as poor self-rated health, misunderstandings about medical conditions and increased mortality risk. ${ }^{20-22}$ However, HL is arguably a broad multidimensional concept that serves as a bridge between literacy skills and abilities and the illness context in which individuals find themselves. ${ }^{23}$ Clearly, some dimensions of literacy skills and abilities are generalisable across all health populations. However, in the presence of a specific illness context, some disease-specific HL would seem necessary for successful self-management of that disease. For example, diabetes-specific HL (DSHL) is particularly salient in the assessment of self-care for type 2 diabetes in adults. ${ }^{24}$ Nevertheless, there is no clear definition of DSHL in the current literature. In general, DSHL represents the ability to obtain and understand diabetes-related information and to make informed diabetes care decisions. A study demonstrated that DSHL was positively associated with self-graded assessment of diabetes care. ${ }^{25}$ Some studies have indicated that DSHL is associated with the diabetes-related knowledge, diabetes-care behaviours and glycaemic control. ${ }^{26} 27$

Thus, pre-diabetes patients with lower levels of DSHL may not have knowledge of the signs or symptoms of concern and may have a higher risk of developing poor health outcomes than those who have higher DSHL.

Several studies have evaluated the impact of HL on HRQoL in patients with type 2 diabetes, ${ }^{28}$ hypertension ${ }^{29}$ and ischaemic heart disease. ${ }^{30}$ However, these studies focus on HL related to obtaining and comprehending general medical information rather than disease-specific or condition-specific HL. Furthermore, some HRQoL measures have also been widely used in cost-utility analyses to determine the cost-effectiveness of treatments and interventions in several populations, including those with chronic conditions. ${ }^{31} 32$ Therefore, an exploration of the impact of DSHL on HRQoL would also be of great importance for determining whether it is necessary to incorporate it as a potential confounding factor in cost-utility analyses of type 2 diabetes interventions. Moreover, examining the association between DSHL and HRQoL could help us to identify new targets and create more precise and multifaceted prevention and intervention strategies to delay the development of type 2 diabetes. At present, there are a few studies that have investigated the relationship between specific HL and HRQoL, and almost no studies in the literature have explored the effect of DSHL on HRQoL among individuals with pre-diabetes.

Therefore, to address these issues and to help to bridge the gap between HL and outcome research in individuals with pre-diabetes, the current study aimed to explore the impact of DSHL on HRQoL among elderly individuals with pre-diabetes in rural areas in China. We hypothesised that elderly individuals with pre-diabetes with high DSHL would report better HRQoL. We hope that this study will contribute to the formulation of effective interventions to improve HRQoL and promote diabetes prevention.

\section{RESEARCH DESIGN AND METHODS \\ Study design}

This cross-sectional study was conducted in the rural areas of Yiyang City of Hunan Province in China between April and July 2015.

\section{Sample size}

The sample size was calculated using the formula for cross-sectional studies, as follows:

$$
N=\frac{Z_{1-/ 2}^{2} p(1-p)}{d^{2}}
$$

where $Z_{1-/ 2}^{2}=1.96$ when $\alpha=0.05$, $p$ is the prevalence of pre-diabetes (which was $20 \%$ in this study according to our presurvey) and $d$ is an admissible error (which was $4 \%$ ). According to the formula, the theoretical sample size was 423 , which included an extra $10 \%$ to allow for subjects lost during the study.

\section{Participants}

Participants in this study were aged 60 years and older and were from the rural areas of Yiyang City of Hunan Province. To select a representative sample of the elderly population with pre-diabetes, a screening programme was carried out among the elderly population in Yiyang City. A multistage cluster randomised sampling method was used to select a representative sample. In the first stage, two (Nanxian and Yuanjiang) out of six counties were selected according to geographical characteristics (north and south of Yiyang City). In the second stage, 2 (Yangluozhou and Yinfengqiao) out of 11 townships from Yuanjiang county and 2 (Qingshuzui and Maocaojie) 
out of 9 townships from Nanxian county were randomly selected by drawn lots. In the third stage, as each township contains 30-50 villages, a proportionate sampling method was used to select $25 \%$ of the villages from each selected township. Thus, 11 villages from Yangluozhou township, 10 villages from Yinfengqiao township, 11 villages from Qingshuzui township and 10 villages from Maocaojie township were randomly selected. In the final stage, all households in each selected village with elderly individuals who had lived in the area for 3 years or longer were eligible to participate in the screening programme $(\mathrm{n}=3197)$. Among them, 603 moved away, 336 had a severe physical or mental illness and 114 refused to participate. Finally, a total of 2144 individuals participated in the screening programme.

An oral glucose tolerance test was used to distinguish between pre-diabetes and normal plasma glucose. The diagnostic standards for pre-diabetes as stated in the 1999 WHO criteria $^{33}$ were (1) an IFG group with fasting plasma glucose of $6.1-7.0 \mathrm{mmol} / \mathrm{L}$ and a 2-hour postglucose load of $<7.8 \mathrm{mmol} / \mathrm{L}$; (2) an IGT group with a 2-hour postglucose load of 7.8-11.1 mmol/L and fasting plasma glucose of $\leq 6.1 \mathrm{mmol} / \mathrm{L}$ and (3) an IFG+IGT group.

More details of the study population and screening procedure have been published elsewhere. ${ }^{34}$ In brief, 2144 elderly individuals took part in the screening programme, and 461 elderly individuals had pre-diabetes. For various reasons, 21 of those with pre-diabetes provided no response, and the response rate was $95.4 \%$. Six individuals who had incomplete data were also excluded from this study. Finally, a total of 434 individuals with pre-diabetes from 42 villages were included in this study.

\section{Data collection}

Sociodemographic information was collected by trained staff using a set of structured questionnaires, which included age, gender, education, marital status, presence of other chronic disease, history of hyperglycaemia, family history of diabetes, physical activity, smoking and alcohol drinking. Marital status was classified as married and non-married. Non-married status included divorced, never married and lost a partner. Chronic diseases included hypertension, coronary heart disease, dyslipidaemia and others. History of hyperglycaemia was defined as a situation of fasting glucose $>6.1 \mathrm{mmol} / \mathrm{L}$ or 2-hour glucose $>7.8 \mathrm{mmol} / \mathrm{L}$ without a diagnosis of diabetes. Physical activity was assessed using the International Physical Activity Questionnaire-long version, and individuals who achieved $\geq 600$ metabolic equivalent-min/week were categorised as active. ${ }^{35}$ Smoking was defined as averaging one or more cigarettes per day in the last year. Alcohol drinking was defined as drinking more than one glass of wine (approximately $250 \mathrm{~mL}$ beer or $100 \mathrm{~mL}$ sake or $20 \mathrm{~mL}$ liquor) per month in the last year.

Anthropometric measurements, including height, weight, blood pressure, waist circumference and hip circumference, were assessed using a standard tool. The measurement procedure was published in a previous study. ${ }^{36}$ Body mass index (BMI) was calculated using the formula of weight in $\mathrm{kg}$ divided by height in $\mathrm{m}^{2}$ $\left(\mathrm{kg} / \mathrm{m}^{2}\right)$. The current Chinese standard classification states that the cut-off values for normal weight, overweight and obesity BMI are $18.5,24.0$ and $28.0 \mathrm{~kg} /$ $\mathrm{m}^{2},{ }^{37}$ respectively. Hypertension was defined as systolic blood pressure $\geq 140 \mathrm{~mm} \mathrm{Hg}$ and/or diastolic blood pressure $\geq 90 \mathrm{~mm} \mathrm{Hg}$. The waist-to-hip ratio (WHR) was calculated by dividing the waist circumference by the hip circumference. A WHR $>0.9$ in men or $>0.8$ in women was defined as abnormal WHR. ${ }^{38}$

DSHL was assessed using the Questionnaire of Health Literacy of Diabetes Mellitus of the Public in China, which was designed by the Chinese Center for Health Education to assess HL about diabetes prevention and control in the general population. ${ }^{39}$ This questionnaire has been widely used in epidemiological studies in China, and has high reliability and validity, with a Cronbach's $\alpha$ of $0.866 .{ }^{39}$ DSHL can provide a comprehensive evaluation of an individual's diabetes prevention and control knowledge, risk awareness and ability to manage risk factors. The questionnaire is organised into three main domains: diabetes-related knowledge, diabetes-related behaviour, and acquisition and utilisation of diabetes information. The diabetes-related knowledge section assessed attitudes towards diabetes, typical symptoms of diabetes, complications of diabetes, factors conferring a high risk of developing diabetes and methods to prevent diabetes. The diabetes-related behaviours included sitting time duration, physical exercise, dietary pattern, physical examination, and smoking and alcohol drinking habits. In the part about the acquisition and utilisation of diabetes information, the participants were asked about the method or way to find diabetes-related information, the degree of their acquisition of diabetes-related information and their ability to identify the correctness of diabetes-related information. An alternative classification was used where the scores 19.5 points and above were classified as high DSHL and the remaining were classified as low. Although the prediabetic population may not experience certain symptoms of diabetes, people with a high HL status can identify the risk factors related to the development of type 2 diabetes, and thus engage in diabetes care behaviour. The purpose and structure of this questionnaire allow it to effectively and accurately measure the participants' ability to obtain, process and understand diabetes-related information and make informed diabetes care decisions.

HRQoL was assessed using the Medical Outcomes Study 36-Item Short-Form Health Survey (SF-36). ${ }^{40}$ The SF-36 health survey questionnaire has been translated and validated in Chinese, and the Chinese version has been proven to be reliable and valid in an elderly population. ${ }^{41}$ This 36-item measure is organised into eight domains that constitute two main components: the physical health 
component and the mental health component. The physical health component includes four parts: physical functioning $(\mathrm{PF})$, role physical $(\mathrm{RP})$, bodily pain $(\mathrm{BP})$ and general health $(\mathrm{GH})$. Vitality (VT), social functioning $(\mathrm{SF})$, role emotional (RE) and mental health $(\mathrm{MH})$ are included in the mental health component. The eight domains were scored from 0 to 100 , indicating the worst to best possible health. Each domain score was further summarised and standardised into the physical health component score (PCS) and the mental health component score (MCS) according to American norms to allow for international comparisons. ${ }^{42}$

\section{Data analysis}

Data were presented as n (\%) for categorical variables and mean \pm SD or median $\left(\mathrm{P}_{25}-\mathrm{P}_{75}\right)$ for numerical variables. Non-parametric tests were used because the distribution of the DSHL scores was non-Gaussian. The MannWhitney U or Kruskal-Wallis test was used to identify the differences in total DSHL scores according to different variables. The t-test or one-way analysis of variance was used to compare the differences in the scores for different domains of HRQoL. General linear models of multivariate analysis of covariance (MANCOVA) were used to test differences in HRQoL between the adequate DSHL and inadequate groups. Sociodemographic and anthropometric variables were treated as possible covariates. A significant MANCOVA was followed by univariate $F$ tests using the Wilks' $\lambda$ statistic. Linear independent pairwise comparisons were analysed to examine the magnitude of the difference in the mean scores of the dependent variables. Effect sizes (ESs) (d) were computed by dividing the difference in means between groups by the pooled SD and were interpreted as small $(\mathrm{d} \leq 0.20)$, medium $(0.20<\mathrm{d} \leq 0.50)$ or large $(0.50<\mathrm{d} \leq 0.80) .{ }^{43}$ The data were analysed using SPSS V.20.0.

\section{Patient and public involvement}

Neither patients nor the public was directly involved in the development, design or recruitment of the study. Anthropometric and glucose test results were provided to the participants at the point of testing.

\section{RESULTS}

A total of 461 elderly individuals had pre-diabetes, and the prevalence of pre-diabetes was $21.5 \%(461 / 2144)$ in rural areas of Yiyang City. In total, 434 elderly individuals with pre-diabetes were included in this study. The average age of all participants was $69.4 \pm 6.4$ years. The average fasting plasma glucose was $5.9 \pm 0.5 \mathrm{mmol} / \mathrm{L}$, and the average 2-hour plasma glucose load was $7.2 \pm 1.9 \mathrm{mmol} / \mathrm{L}$. A majority of the subjects were female, had completed less than 6 years of education, smoked, drank no alcohol and had no hypertension. The characteristics of the study subjects are presented in table 1.

The overall median DSHL score was 10.0 (IQR 7.0-13.0). Men had lower HL scores than women. Furthermore,
Table 1 The DSHL score according to different characteristics

\begin{tabular}{|c|c|c|c|}
\hline Characteristics & n (\%) & DSHL score* & $\mathbf{P}$ value \\
\hline \multicolumn{4}{|l|}{ Age } \\
\hline 60-69 years & $239(55.1)$ & $10.0(8.0-15.0)$ & 0.461 \\
\hline 70 years and older & $195(44.9)$ & $10.0(7.5-11.0)$ & \\
\hline \multicolumn{4}{|l|}{ Gender } \\
\hline Male & $180(41.5)$ & $9.0(7.0-12.0)$ & $<0.001$ \\
\hline Female & $254(58.5)$ & $11.0(8.0-13.0)$ & \\
\hline \multicolumn{4}{|l|}{ Marital status } \\
\hline Married & $312(71.9)$ & $10.0(7.0-13.0)$ & 0.044 \\
\hline Non-married & $122(28.1)$ & $9.0(7.0-11.0)$ & \\
\hline \multicolumn{4}{|l|}{ Education } \\
\hline Less than 6 years & $353(81.3)$ & $9.0(6.5-12.0)$ & $<0.001$ \\
\hline 6 years and more & $81(18.7)$ & $12.0(9.0-16.0)$ & \\
\hline \multicolumn{4}{|c|}{ History of hyperglycaemia } \\
\hline Yes & $28(6.5)$ & $12.5(9.3-20.5)$ & 0.001 \\
\hline No & 406 (93.5) & $9.0(7.0-12.0)$ & \\
\hline \multicolumn{4}{|c|}{ Family history of diabetes } \\
\hline Yes & $36(8.3)$ & $12.0(7.0-13.8)$ & 0.165 \\
\hline No & $398(91.7)$ & $10.0(7.0-12.0)$ & \\
\hline \multicolumn{4}{|l|}{$\begin{array}{l}\text { Have other chronic } \\
\text { disease }\end{array}$} \\
\hline Yes & $176(40.6)$ & $10.0(7.0-13.0)$ & 0.544 \\
\hline No & $258(59.4)$ & $10.0(7.0-13.0)$ & \\
\hline \multicolumn{4}{|l|}{ Physical activity } \\
\hline Active & $182(41.9)$ & $10.5(8.0-13.5)$ & 0.227 \\
\hline Inactive & $252(58.1)$ & $9.5(8.0-13.0)$ & \\
\hline \multicolumn{4}{|l|}{ Smoking } \\
\hline Yes & $99(22.8)$ & $10.0(8.0-12.0)$ & 0.525 \\
\hline No & $335(77.2)$ & $10.0(8.0-13.0)$ & \\
\hline \multicolumn{4}{|l|}{ Alcohol drinking } \\
\hline Yes & $98(22.6)$ & $10.0(8.0-12.0)$ & 0.308 \\
\hline No & $336(77.4)$ & $10.0(7.5-13.0)$ & \\
\hline \multicolumn{4}{|l|}{ BMI } \\
\hline Lean & $17(3.9)$ & $9.0(5.5-13.5)$ & 0.547 \\
\hline Normal & $233(53.7)$ & $9.0(7.0-13.0)$ & \\
\hline Overweight & $129(29.7)$ & $10.0(7.0-12.0)$ & \\
\hline Obese & 55 (12.7) & $10.0(7.0-13.0)$ & \\
\hline \multicolumn{4}{|l|}{ Hypertension } \\
\hline Yes & 173 (39.9) & $10.5(8.5-13.0)$ & 0.256 \\
\hline No & $261(60.1)$ & $9.5(8.0-12.0)$ & \\
\hline \multicolumn{4}{|l|}{ WHR } \\
\hline Normal & 77 (17.7) & $9.0(7.0-12.0)$ & 0.074 \\
\hline Abnormal & $357(82.3)$ & $10.0(7.0-13.0)$ & \\
\hline
\end{tabular}

*Data are presented as the median $\left(\mathrm{P}_{25}-\mathrm{P}_{75}\right)$.

†P value was determined by Kruskal-Wallis or Mann-Whitney $U$ test. $\mathrm{BMI}$, body mass index; DSHL, diabetes-specific health literacy; WHR, waist-to-hip ratio.

married elderly individuals had higher DSHL scores than non-married individuals. Individuals with a history of hyperglycaemia had a higher DSHL score than people with no history. Similarly, individuals with pre-diabetes 
who had completed 6 years or more of education had a higher score than those who had completed less than 6 years. The DSHL score according to different characteristics is presented in table 1 .

\section{HRQOL score}

Individuals with pre-diabetes reported a PCS of 42.1 points (95\% CI 41.2 to 43.1 ) and an MCS of 46.4 points (95\% CI 45.5 to 47.1). The scores for the four domains of the PCS were $76.1 \pm 23.4,71.4 \pm 42.4,75.7 \pm 15.9$ and $57.8 \pm 21.5$, respectively, and the MCS were $72.2 \pm 18.1,79.7 \pm 17.1,85.1 \pm 33.3$ and $74.8 \pm 17.5$, respectively. The means and their SDs for eight subscales of HRQoL scores according to different characteristics are presented in table 2. Neither domain score showed a significant difference for the variables of gender, family history of diabetes or alcohol drinking (all $\mathrm{p}>0.05$ ). The BP and GH scores were lower among people aged 70 years and older. The MH score was lower among people who were not married. Individuals with pre-diabetes who had completed 6 years of education or more had higher SF and RE scores than people educated 1-6 years. Individuals who achieved active physical activity seemed to have higher scores in the PF, BP and GH domains. The RP, GH and RE scores were similarly higher among elderly people with normal BMI. Moreover, individuals with normal WHR had higher BP, SF and RE scores.

\section{Association between DSHL and HRQOL}

Crude analysis indicated that when the eight subscales of HRQoL were placed as the dependent variables and DSHL (as a binary variable) was entered as the independent variable, the overall MANCOVA showed significant differences in the GH, VT, SF and MH scores between the two groups (Wilks' $\lambda=0.955, \mathrm{~F}=2.44, \mathrm{p}=0.014$ ). After adjusting for other covariants, individuals with high DSHL reported higher scores on $\mathrm{GH}\left(\mathrm{M}_{\text {diff }}=6.8, \mathrm{p}=0.028\right)$, VT $\left(\mathrm{M}_{\text {diff }}=6.6, \mathrm{p}=0.014\right), \mathrm{SF}\left(\mathrm{M}_{\text {diff }}=6.0, \mathrm{p}=0.017\right)$ and $\mathrm{MH}$ $\left(\mathrm{M}_{\text {diff }}=7.4, \mathrm{p}=0.005\right)$ than did those with low DSHL. The associations between DSHL and different domains of HRQoL are presented in table 3.

Crude analysis showed that with two components of HRQoL entered as dependent variables, the overall MANCOVA was significant (Wilks' $\lambda=0.965, \quad \mathrm{~F}=7.87$, $\mathrm{p}<0.001)$. Individuals with high DSHL had higher PCS $\left(\mathrm{M}_{\text {diff }}=2.9, \mathrm{ES}=0.30\right)$ and $\mathrm{MCS}\left(\mathrm{M}_{\text {diff }}=4.4, \mathrm{ES}=0.47\right)$ than those with low DSHL. After adjusting for age, gender, education, marital status, other chronic disease, family history of diabetes, history of hyperglycaemia, physical activity, hypertension, smoking, drinking, BMI and WHR, a linear independent pairwise comparison indicated that individuals with pre-diabetes who had higher DSHL reported higher MCS $\left(\mathrm{M}_{\text {diff }}=3.5,95 \%\right.$ CI 1.8 to 6.3$)$ with a medium ES (ES=0.38). The association between DSHL and HRQoL among elderly individuals with pre-diabetes is presented in table 4 .

\section{DISCUSSION}

This cross-sectional study showed a high prevalence $(21.5 \%)$ of pre-diabetes among the elderly population in rural areas in China, which is similar to the findings of the earlier study. ${ }^{44}$ The results, together with the large elderly population living in rural areas, suggest that this serious public health problem in China requires better prevention.

Many studies have used general HL measurement instruments, such as The Rapid Estimate of Adult Literacy in Medicine(REALM) or The Test of Functional Health Literacy in Adults(TOFHLA), which are not disease-specific or condition-specific. However, our study used a DSHL questionnaire with high reliability and validity that was designed by the Chinese Center for Health Education, and is suitable for a non-diabetic population. ${ }^{39}$ The questionnaire was able to effectively and accurately examine the level of HL about diabetes knowledge, diabetes prevention behaviours, and the acquisition and utilisation of diabetes information among individuals with pre-diabetes. There is a direct association between DSHL and patient assessments of their self-care ability, which indicates that HL measures should include indicators of knowledge and understanding. ${ }^{25}$ Thus, in terms of prevention, knowing the HL of individuals with pre-diabetes regarding diabetes prevention and control contribute to the development of more effective interventions and health education methods. Based on the results of the univariate analysis, the DSHL score showed significant differences in the variables of gender, education and history of hyperglycaemia, which are consistent with the findings of other studies. 4546

Although the effect of HL on HRQoL has been widely discussed among some populations in previous studies, ${ }^{47-49}$ few studies have explored the association between HL and HRQoL among individuals with pre-diabetes. There is also a lack of research probing the effect of disease-specific or condition-specific HL on HRQoL. To the best of our knowledge, this is the first study to examine the relationship between DSHL and HRQoL among elderly individuals with pre-diabetes. Our study found that DSHL was positively associated with some health domains of HRQoL according to bivariate and multivariate analyses. Compared with individuals with pre-diabetes with lower HL levels, subjects with higher HL reported higher scores on the GH, VT, SF and MH subscales of HRQoL. That is, prediabetic older adults with lower HL were more likely to have limited social activities $(\mathrm{SF})$, poor general health perceptions $(\mathrm{GH})$, tiredness (VT) and psychological distress (MH). When the eight domain scores were standardised and summarised as the PCS and MCS, the relationship between DSHL levels and HRQoL was significant in the mental wellbeing (SF-36 MCS), while it was significant in the physical health domain (SF-36 PCS) only in the bivariate model and became non-significant after controlling for sociodemographic and somatometric covariates. On one hand, more subscales of the MCS component than of the PCS were significantly associated with DSHL; this finding could be helpful in further studies exploring the influence of HL on certain subscales of HRQoL. On the other 
Table 2 HRQoL scores of eight domains measured by SF-36

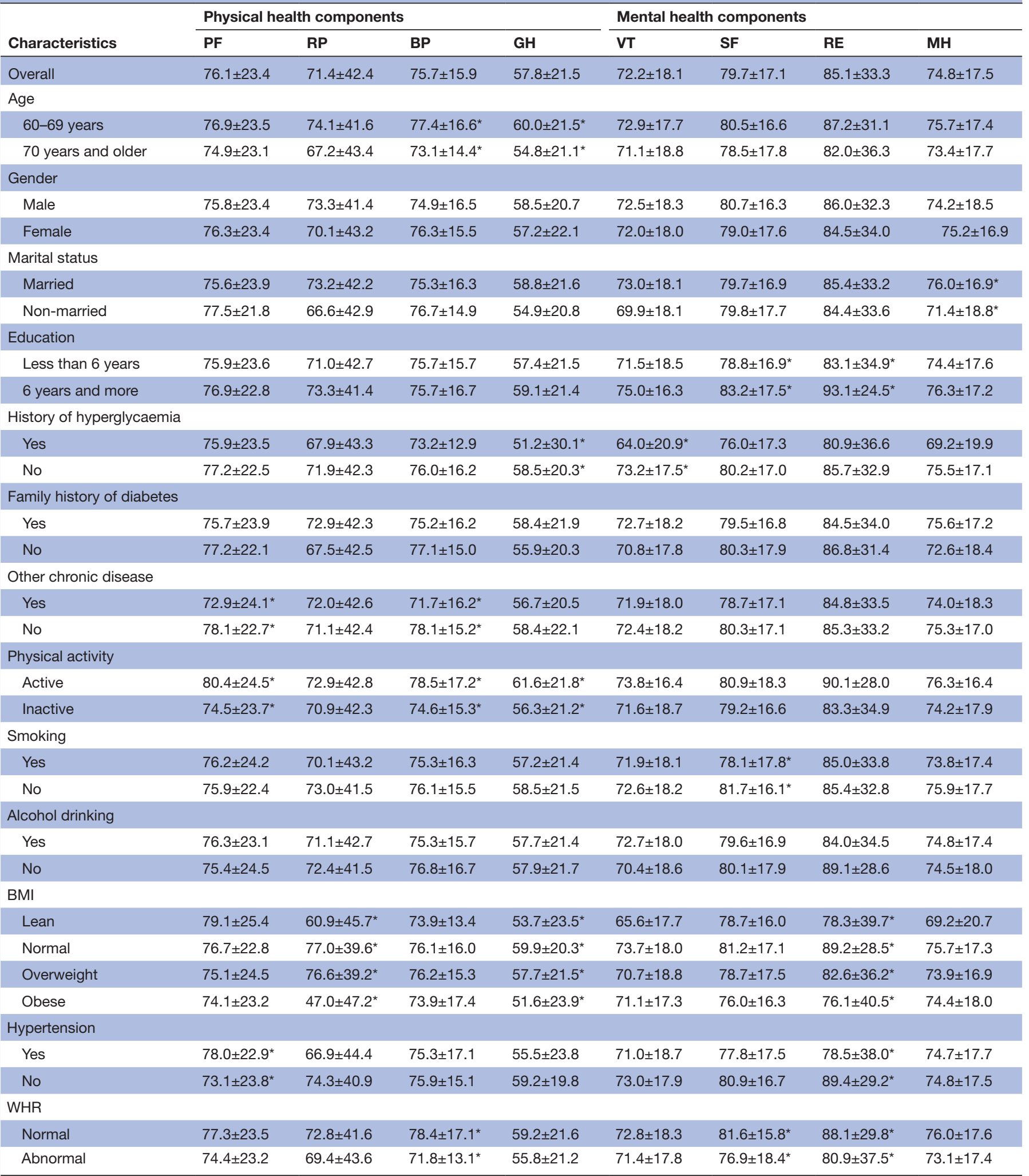

Data are presented as the mean $\pm \mathrm{SD}$, and analysis was performed using the analysis of variance or t-test. ${ }^{\star} \mathrm{P}<0.05$.

BMI, body mass index; BP, bodily pain; GH, general health; HRQoL, health-related quality of life; MH, mental health; PF, physical functioning; RE, role emotional; RP, role physical; SF, social functioning; SF-36, Medical Outcomes Study 36-Item Short-Form Health Survey; VT, vitality; WHR, waist-to-hip ratio.

hand, some information loss may occur in the process of standardising and summarising the scores of the eight domains into two components because of the different weights of the eight domains. However, the PCS and MCS scales are scored using the linear T-score transformation method so that a one-point difference is one-tenth of a 
Table 3 Association between DSHL and different subscales of HRQoL among elderly individuals with pre-diabetes

\begin{tabular}{|c|c|c|c|c|c|c|c|}
\hline \multirow[b]{2}{*}{ SF-36 domains } & \multicolumn{2}{|c|}{ High DSHL } & \multicolumn{2}{|c|}{ Low DSHL } & \multicolumn{3}{|l|}{ Difference } \\
\hline & Mean & SE & Mean & SE & $M_{\text {diff }}(95 \% \mathrm{Cl})$ & ES (d) & $P$ value \\
\hline \multicolumn{8}{|c|}{ Crude analysis (Wilks' $\lambda=0.955, F=2.44, p=0.014$ ) } \\
\hline $\mathrm{RP}$ & 74.5 & 5.8 & 70.9 & 2.3 & $3.5(-5.7$ to 15.4$)$ & 0.08 & 0.224 \\
\hline $\mathrm{BP}$ & 78.9 & 2.2 & 75.2 & 0.8 & $3.7(-1.8$ to 8.3$)$ & 0.23 & 0.110 \\
\hline VT & 78.9 & 2.5 & 71.3 & 0.9 & 7.5 (2.4 to 12.8$)$ & 0.42 & 0.004 \\
\hline SF & 86.0 & 2.3 & 78.8 & 0.9 & 7.2 (2.4 to 12.1$)$ & 0.43 & 0.001 \\
\hline RE & 91.2 & 4.6 & 84.3 & 1.7 & $6.9(-2.7$ to 16.5$)$ & 0.21 & 0.158 \\
\hline $\mathrm{MH}$ & 81.8 & 2.4 & 73.8 & 0.9 & 8.0 (3.0 to 13.0 ) & 0.46 & 0.002 \\
\hline \multicolumn{8}{|c|}{ Adjusted analysis (Wilks' $\lambda=0.958, F=2.31, p=0.019)^{\star}$} \\
\hline $\mathrm{BP}$ & 78.4 & 2.1 & 75.3 & 0.8 & $3.1(-1.2$ to 7.5$)$ & 0.19 & 0.161 \\
\hline $\mathrm{GH}$ & 63.7 & 2.9 & 56.9 & 1.1 & 6.8 (1.7 to 12.9$)$ & 0.33 & 0.028 \\
\hline VT & 78.0 & 2.5 & 71.4 & 0.9 & 6.6 (1.3 to 11.8$)$ & 0.37 & 0.014 \\
\hline SF & 84.9 & 2.3 & 79.0 & 0.9 & 6.0 (1.1 to 10.9$)$ & 0.36 & 0.017 \\
\hline $\mathrm{RE}$ & 88.0 & 4.6 & 84.7 & 1.7 & $3.4(-6.2$ to 12.9$)$ & 0.10 & 0.492 \\
\hline $\mathrm{MH}$ & 81.2 & 2.4 & 73.9 & 0.9 & 7.4 (2.3 to 12.5$)$ & 0.43 & 0.005 \\
\hline
\end{tabular}

${ }^{*}$ Adjusted for age, gender, education, marital status, other chronic disease, physical activity, family history of diabetes, history of hyperglycaemia, smoking, drinking, hypertension, BMI and WHR.

BMI, body mass index; BP, bodily pain; DSHL, diabetes-specific health literacy; ES (d), effect size (mean difference/pooled SD); GH, general health; HRQoL, health-related quality of life; $\mathrm{M}_{\text {diff }}$ mean difference; $\mathrm{MH}$, mental health; PF, physical functioning; RE, role emotional; RP, role physical; SF, social functioning; SF-36, Medical Outcomes Study 36-Item Short-Form Health Survey; VT, vitality; WHR, waist-to-hip ratio.

$\mathrm{SD}$, and higher scores indicate a better health status. ${ }^{42}$ Therefore, a two-point to three-point difference in the PCS and MCS in our study is significant and meaningful. These results are in concordance with those of previous studies that targeted the relationship between general HL and HRQoL. ${ }^{10} 50-53$ For instance, Jayasinghe et al found that HL accounted for $45 \%$ and $70 \%$ of the total between-patient variance explained in PCS-12 and MCS-12, respectively. ${ }^{10}$ Furthermore, a study conducted in 605 patients with symptomatic heart failure showed that those with higher literacy had better HRQoL scores (mean difference $=7.2, \mathrm{p}<0.01$ ) than did those with lower literacy. ${ }^{52}$ A cross-sectional survey of 1841 cancer patients in Wisconsin also indicated that higher HL was positively associated with the physical, functional, emotional and social well-being subscales of HRQoL. ${ }^{53}$ However, our results also contradict the findings of previous studies that examined the association. ${ }^{28-30} 54-56$ Data from a clinical

Table 4 Association between DSHL and HRQoL among elderly individuals with pre-diabetes

\begin{tabular}{|c|c|c|c|c|c|c|c|}
\hline \multirow[b]{2}{*}{ Variables } & \multicolumn{2}{|c|}{ High DSHL } & \multicolumn{2}{|c|}{ Low DSHL } & \multicolumn{3}{|l|}{ Difference } \\
\hline & Mean & SE & Mean & SE & $M_{\text {diff }}(95 \% \mathrm{Cl})$ & ES (d) & $P$ value \\
\hline \multicolumn{8}{|c|}{ Crude analysis (Wilks' $\lambda=0.965, F=7.87, p<0.001$ ) } \\
\hline MCS & 50.2 & 1.3 & 45.8 & 0.5 & $4.4(1.7$ to 7.1$)$ & 0.47 & 0.001 \\
\hline \multicolumn{8}{|c|}{ Adjusted analysis (Wilks' $\lambda=0.974, F=5.63, p=0.004)^{\star}$} \\
\hline MCS & 49.4 & 1.3 & 45.9 & 0.7 & 3.5 (1.8 to 6.3$)$ & 0.38 & 0.012 \\
\hline
\end{tabular}

*Adjusted for age, gender, education, marital status, other chronic disease, physical activity, family history of diabetes, history of hyperglycaemia, smoking, drinking, hypertension, BMI and WHR.

BMI, body mass index; DSHL, diabetes-specific health literacy; ES (d), effect size (mean difference/pooled SD); HRQoL, health-related quality of life; MCS, mental health component score; $\mathrm{M}_{\text {diff, }}$ mean difference; PCS, physical health component score; WHR, waist-to-hip ratio. 
trial that included 154 predominantly white patients with type 2 diabetes who screened positive for depression showed that the between-HL group difference in change over 1 year was only non-significant at 0.76 points for PCS and 0.56 points for MCS. ${ }^{28}$ In another study conducted among frequent users of healthcare services, no association was found between HL and HRQoL on both PCS and MCS. ${ }^{55}$ Two other studies ${ }^{3054}$ demonstrated that HL was not significantly associated with the mental component of HRQoL. A prospective cohort study of 4278 older adults in the UK showed that low HL significantly predicted declines in the physical, psychological and environmental domains of HRQoL but not in the social relationship HRQoL. ${ }^{56}$ There are three reasons for this variance. First, most studies pay attention to the impact of general HL rather than specific HL on HRQoL. However, general HL includes the ability to obtain, process and understand all basic health information, not just a specific disease. Second, the various studies used different tools to measure HL and HRQoL. Last, the contradictory results were also likely due to differences in social and cultural factors, and in the study populations and sample sizes.

These results suggest that individuals with newly diagnosed pre-diabetes who have higher levels of DSHL may have higher HRQoL, especially for the mental health component. A potential explanation for the relationship between DSHL and the physical and mental components of HRQoL may be that low DSHL limits individuals' understanding of complex information about diabetes knowledge and prevention, and thus becomes a barrier to individuals' participation in diabetes education and intervention. Moreover, people with lower HL tend to have difficulty communicating, which prevents them from not only asking questions, but also clearly expressing their concerns, emotions, and needs to providers as well as seeking additional services, such as support for mental health. ${ }^{50}{ }^{53}$ Furthermore, a previous study found that subjects with low HL were three times more likely to have depression. ${ }^{54}$ Considering that individuals with lower HL were more likely to have limited social activities, tiredness and psychological distress, lower DSHL may further limit individuals' ability to talk with their families and health education and care providers about difficult emotional issues or abstract psychosocial implications of diabetes. Thus, different DSHL groups may show differences in understanding and acceptance when faced with the same diabetes education information and intervention programmes. This process may be also associated with responsiveness during consultations and interventions. Individuals with lower levels of DSHL may not have knowledge of signs or symptoms of concern and may experience a psychological panic, reducing the MCS of HRQoL. The findings about the impact of DSHL on HRQoL in the prediabetic population could help us to identify new target groups and provide multifaceted and collaborative interventions to delay the development of type 2 diabetes. They also provide information that could contribute to assessments of the effects and cost-effectiveness of diabetes education and intervention. Healthcare staff should be aware of HL problems among elderly adults, and should simplify health-related information to increase the responsiveness of subjects with low HL during consultations and interventions. Although our findings were based on the results of a cross-sectional study, HRQoL could be viewed as an essential supplementary outcome in health surveys or intervention process; thus, it is important to carry out HRQoL monitoring to fully understand the health status of different HL groups. Furthermore, the finding that DSHL is associated with changes in HRQoL outcomes raises the need for testing the hypothesis of whether DSHL is a modifiable factor and, if so, considering whether interventions aimed at improving DSHL may also lead to improvements in HRQoL and health conditions in this population. Therefore, there are important public health implications of examining the association between DSHL and HRQoL.

Our study also revealed that individuals with pre-diabetes showed lower PCS than MCS, and the mean scores of the four domains of the mental health components were likewise higher than those of four subscales of the physical health components, which was consistent with the findings of other studies. ${ }^{5758}$ One explanation is that some elderly have difficulties in physical activities due to illness. A study has also shown that chronic diseases have a stronger effect on reducing physical function than psychological function. ${ }^{59}$ Similar to the results of our study, elderly individuals with chronic disease, overweight or obesity and physical inactivity have lower scores on the subscales of physical function, $\mathrm{BP}$ and GH; however, these domains are components of the physical health aspect of HRQoL.

Our study also has several limitations. First, its cross-sectional design did not permit causal inferences. Furthermore, both cohort studies and randomised controlled trial designs garner a deeper understanding of the relationship between DSHL and HRQoL. Second, HL was measured using the public questionnaire of HL of diabetes mellitus. This may influence the way in which our study may be compared with previous studies, the majority of which measured multidimensional competences rather than a single competence of functional HL. Third, self-administered questionnaires were used to assess some variables, which might have introduced recall bias. However, this limitation was minimised because the instruments used in this study are valid and reliable. Finally, our study sample was taken from rural areas in one city of one province of China. Therefore, the generalisation of the results to other populations should be carefully considered.

\section{CONCLUSIONS}

In summary, lower DSHL was associated with poorer HRQoL among elderly individuals with pre-diabetes in rural areas in China, particularly in terms of the mental health component. These findings suggest that assessing 
and improving both DSHL and HRQoL may be important for individuals with pre-diabetes.

Acknowledgements We thank all the participants very much for their collaboration.

Contributors $\mathrm{ZH}$ and $\mathrm{LQ}$ completed the statistical analyses and drafted the manuscript. HX checked and revised the manuscript. All the authors read and approved the final manuscript.

Funding This study was funded by the Teachers Research Fund of Central South University (2013JSJJ034) and the Central South University Graduate Student Independent Exploration Innovation Project (No. 2013zzts286).

Competing interests None declared.

Patient consent for publication Obtained.

Ethics approval The study was approved by the Medical Ethics Committee of Central South University (Changsha, China; Identification code: CTXY-150002-7; 27 February 2015).

Provenance and peer review Not commissioned; externally peer reviewed.

Data availability statement The data analysed during this study are included in the article. The numerical data used to support the findings of this study are available from the corresponding author upon reasonable request.

Open access This is an open access article distributed in accordance with the Creative Commons Attribution Non Commercial (CC BY-NC 4.0) license, which permits others to distribute, remix, adapt, build upon this work non-commercially, and license their derivative works on different terms, provided the original work is properly cited, appropriate credit is given, any changes made indicated, and the use is non-commercial. See: http://creativecommons.org/licenses/by-nc/4.0/.

\section{REFERENCES}

1. Punthakee Z, Goldenberg R, Katz P, et al. Definition, classification and diagnosis of diabetes, prediabetes and metabolic syndrome. Can J Diabetes 2018;42 Suppl 1:S10-S15.

2. Perreault L, Pan Q, Mather KJ, et al. Effect of regression from prediabetes to normal glucose regulation on long-term reduction in diabetes risk: results from the diabetes prevention program outcomes study. Lancet 2012;379:2243-51.

3. de Vegt F, Dekker JM, Jager A, et al. Relation of impaired fasting and postload glucose with incident type 2 diabetes in a Dutch population: the Hoorn study. JAMA 2001;285:2109-13.

4. Ang YG, WU CX, Toh MPHS, et al. Progression rate of newly diagnosed impaired fasting glycemia to type 2 diabetes mellitus: a study using the National healthcare group diabetes registry in Singapore. J Diabetes 2012;4:159-63.

5. Forouhi NG, Luan J, Hennings S, et al. Incidence of type 2 diabetes in England and its association with baseline impaired fasting glucose: the Ely study 1990-2000. Diabet Med 2007;24:200-7.

6. Nathan DM, Davidson MB, DeFronzo RA, et al. Impaired fasting glucose and impaired glucose tolerance: implications for care. Diabetes Care 2007;30:753-9.

7. Wang L, Gao P, Zhang M, et al. Prevalence and ethnic pattern of diabetes and prediabetes in China in 2013. JAMA 2017;317:2515-23.

8. The WHOQOL Group. The world Health organization quality of life assessment (WHOQOL): development and general psychometric properties. Soc Sci Med 1998;46:1569-85.

9. Guyatt GH, Feeny DH, Patrick DL. Measuring health-related quality of life. Ann Intern Med 1993;118:622-9.

10. Jayasinghe UW, Harris MF, Parker SM, et al. The impact of health literacy and life style risk factors on health-related quality of life of Australian patients. Health Qual Life Outcomes 2016;14:68.

11. Keles H, Ekici A, Ekici M, et al. Effect of chronic diseases and associated psychological distress on health-related quality of life. Intern Med J 2007;37:6-11.

12. Alfonso-Rosa RM, Del Pozo-Cruz B, Del Pozo-Cruz J, et al. The relationship between nutritional status, functional capacity, and health-related quality of life in older adults with type 2 diabetes: a pilot explanatory study. J Nutr Health Aging 2013;17:315-21.

13. Tapp RJ, Dunstan DW, Phillips P, et al. Association between impaired glucose metabolism and quality of life: results from the Australian diabetes obesity and lifestyle study. Diabetes Res Clin Pract 2006;74:154-61.

14. Ghorbani A, Ziaee A, Esmailzadehha N, et al. Association between health-related quality of life and impaired glucose metabolism in Iran: the Qazvin metabolic diseases study. Diabet Med 2014;31:754-8.
15. Hunger M, Holle R, Meisinger $\mathrm{C}$, et al. Longitudinal changes in health-related quality of life in normal glucose tolerance, prediabetes and type 2 diabetes: results from the KorA S4/F4 cohort study. Qual Life Res 2014;23:2515-20.

16. Lam CLK, Fong DYT, Lauder IJ, et al. The effect of health-related quality of life (HRQOL) on health service utilisation of a Chinese population. Soc Sci Med 2002;55:1635-46.

17. Chen T, Li L. Influence of health-related quality of life on health service utilization in addition to socio-demographic and morbidity variables among primary care patients in China. Int J Public Health 2009;54:325-32.

18. Alonso J, Ferrer M, Gandek B, et al. Health-Related quality of life associated with chronic conditions in eight countries: results from the International quality of life assessment (IQOLA) project. Qual Life Res 2004;13:283-98.

19. Berkman ND, Davis TC, McCormack L. Health literacy: what is it? J Health Commun 2010;15 Suppl 2:9-19.

20. Gazmararian JA, Williams MV, Peel J, et al. Health literacy and knowledge of chronic disease. Patient Educ Couns 2003;51:267-75.

21. Bennett IM, Chen J, Soroui JS, et al. The contribution of health literacy to disparities in self-rated health status and preventive health behaviors in older adults. Ann Fam Med 2009;7:204-11.

22. Bostock S, Steptoe A. Association between low functional health literacy and mortality in older adults: longitudinal cohort study. BMJ 2012;344:e1602.

23. Institute of Medicine Committee on Health Literacy, NielsenBohlman L, Panzer AM, Kindig DA, eds. Health literacy: a prescription to end confusion. Washington, DC: National Academies Press (US) Copyright 2004 by the National Academy of Sciences, 2004.

24. Norris SL, Lau J, Smith SJ, et al. Self-Management education for adults with type 2 diabetes: a meta-analysis of the effect on glycemic control. Diabetes Care 2002;25:1159-71.

25. Yamashita T, Kart CS. Is diabetes-specific health literacy associated with diabetes-related outcomes in older adults? J Diabetes 2011;3:138-46.

26. Chen G-D, Huang C-N, Yang Y-S, et al. Patient perception of understanding health education and instructions has moderating effect on glycemic control. BMC Public Health 2014;14:683.

27. Sarkar U, Fisher L, Schillinger D. Is self-efficacy associated with diabetes self-management across race/ethnicity and health literacy? Diabetes Care 2006;29:823-9.

28. Al Sayah F, Majumdar SR, Johnson JA. Association of inadequate health literacy with health outcomes in patients with type 2 diabetes and depression: secondary analysis of a controlled trial. Can $J$ Diabetes 2015;39:259-65.

29. Naimi AJ, Naderiravesh N, Bayat ZS, et al. Correlation between health literacy and health-related quality of life in patients with hypertension, in Tehran, Iran, 2015-2016. Electron Physician 2017;9:5712-20.

30. González-Chica DA, Mnisi Z, Avery J, et al. Effect of health literacy on quality of life amongst patients with ischaemic heart disease in Australian general practice. PLoS One 2016;11:e0151079.

31. Robinson R. Cost-Utility analysis. BMJ 1993;307:859-62.

32. Zhang X-H, Li S-C, Fong K-Y, et al. The impact of health literacy on health-related quality of life (HRQoL) and utility assessment among patients with rheumatic diseases. Value Health 2009;12 Suppl 3:S106-S109.

33. Alberti KG, Zimmet PZ. Definition, diagnosis and classification of diabetes mellitus and its complications. Part 1: diagnosis and classification of diabetes mellitus provisional report of a who consultation. Diabet Med 1998;15:539-53.

34. Xu H, Tang L, Hu Z, et al. Association between physical activity and health-related quality of life in elderly individuals with pre-diabetes in rural Hunan Province, China: a cross-sectional study. BMJ Open 2018;8:e019836.

35. Pate RR, Pratt M, Blair SN, et al. Physical activity and public health. A recommendation from the centers for disease control and prevention and the American College of sports medicine. JAMA 1995;273:402-7.

36. Qin L, Xu H. A cross-sectional study of the effect of health literacy on diabetes prevention and control among elderly individuals with prediabetes in rural China. BMJ Open 2016;6:e011077.

37. Wu Y. Overweight and obesity in China. BMJ 2006;333:362-3.

38. Obesity in Asia Collaboration. Is central obesity a better discriminator of the risk of hypertension than body mass index in ethnically diverse populations? J Hypertens 2008;26:169-77.

39. Li L, Li Y, Nie X, et al. [An analysis of health literacy about diabetes prevention and control and its influencing factors among the residents in six provinces in China]. Zhonghua Yu Fang Yi Xue Za Zhi 2014:48:561-5. 
40. Ware JE, Sherbourne CD. The MOS 36-item short-form health survey (SF-36). I. conceptual framework and item selection. Med Care 1992;30:473-83.

41. Zhou B, Chen K, Wang J-fang, et al. [Reliability and validity of a short-form health survey scale (SF-36), Chinese version used in an elderly population of Zhejiang province in China]. Zhonghua Liu Xing Bing Xue Za Zhi 2008;29:1193-8.

42. Ware J, Kosinski M, Keller S. SF-36 physical and mental health summary scales: a user's manual. 5th edn. Boston, MA: Health Assessment Lab. New England Medical Center, 1994.

43. Cohen J. A power primer. Psychol Bull 1992;112:155-9.

44. Xu Y, Wang L, He J, et al. Prevalence and control of diabetes in Chinese adults. JAMA 2013;310:948-59.

45. Lutfiyya MN, Lipsky MS, Bales RW, et al. Disparities in knowledge of heart attack and stroke symptoms among adult men: an analysis of behavioral risk factor surveillance survey data. $J$ Natl Med Assoc 2008;100:1116-24.

46. Aihara Y, Minai J. Barriers and catalysts of nutrition literacy among elderly Japanese people. Health Promot Int 2011;26:421-31.

47. Miller DB, Cage JL, Nowacki AS, et al. Health Literacy $(\mathrm{HL})$ \& HealthRelated Quality of Life (HRQL) Among Minority Men. J Natl Med Assoc 2018;110:124-9.

48. Montbleau KE, King D, Henault L, et al. Health literacy, health-related quality of life, and atrial fbrillation. Cogent Med 2017;4.

49. Divaris K, Lee JY, Baker AD, et al. The relationship of oral health literacy with oral health-related quality of life in a multi-racial sample of low-income female caregivers. Health Qual Life Outcomes 2011:9:108.

50. Song L, Mishel M, Bensen JT, et al. How does health literacy affect quality of life among men with newly diagnosed clinically localized prostate cancer? Flndings from the North Carolina-Louisiana prostate cancer project (PCaP). Cancer 2012;118:3842-51.
51. Sayah FA, Qiu W, Johnson JA. Health literacy and health-related quality of life in adults with type 2 diabetes: a longitudinal study. Qual Life Res 2016;25:1487-94.

52. Macabasco-O'Connell A, DeWalt DA, Broucksou KA, et al. Relationship between literacy, knowledge, self-care behaviors, and heart failure-related quality of life among patients with heart failure. $J$ Gen Intern Med 2011;26:979-86.

53. Halverson JL, Martinez-Donate AP, Palta M, et al. Health literacy and health-related quality of life among a population-based sample of cancer patients. J Health Commun 2015;20:1320-9.

54. Lincoln A, Paasche-Orlow MK, Cheng DM, et al. Impact of health literacy on depressive symptoms and mental health-related quality of life among adults with addiction. J Gen Intern Med 2006;21:818-22.

55. Couture Éva Marjorie, Chouinard M-C, Fortin M, et al. The relationship between health literacy and quality of life among frequent users of health care services: a cross-sectional study. Health Qual Life Outcomes 2017;15:137.

56. Panagioti M, Skevington SM, Hann M, et al. Effect of health literacy on the quality of life of older patients with long-term conditions: a large cohort study in UK general practice. Qual Life Res 2018;27:1257-68.

57. Taylor LM, Spence JC, Raine K, et al. Physical activity and healthrelated quality of life in individuals with prediabetes. Diabetes Res Clin Pract 2010;90:15-21.

58. Ibrahim N, Moy FM, Awalludin IAN, et al. The health-related quality of life among pre-diabetics and its association with body mass index and physical activity in a semi-urban community in Malaysia--a cross sectional study. BMC Public Health 2014;14:298.

59. Cigolle CT, Langa KM, Kabeto MU, et al. Geriatric conditions and disability: the health and retirement study. Ann Intern Med 2007;147:156-64. 